\title{
Key Role of Mitochondrial Mutation Leu107Ser (COX1) in Deltamethrin Resistance in Salmon Lice (Lepeophtheirus Salmonis)
}

Claudia Tschesche

University of Stirling

Michaël Bekaert

University of Stirling

David I. Bassett

University of Stirling

Sally Boyd

University of Stirling

James E. Bron

University of Stirling

Armin Sturm ( $\nabla$ armin.sturm@stir.ac.uk)

University of Stirling

\section{Research Article}

Keywords: Sea lice, Caligidae, Mitochondria, Parallel evolution, Aquaculture, Atlantic salmon

Posted Date: October 8th, 2021

DOI: https://doi.org/10.21203/rs.3.rs-953014/v1

License: (1) This work is licensed under a Creative Commons Attribution 4.0 International License.

Read Full License 


\section{Abstract}

Deltamethrin (DTM) is used to treat Atlantic salmon (Salmon salar) against salmon lice (Lepeophtheirus salmonis) infestations. However, development of DTM resistance has been reported from North Atlantic L. salmonis populations, in which resistance is associated with mitochondrial (mtDNA) mutations. This study investigated the relationship between DTM resistance and mtDNA single nucleotide polymorphisms (SNPs). A total of $188 \mathrm{~L}$. salmonis collected from Scottish aquaculture sites were assessed using DTM bioassays and genotyped at 18 SNP loci. Genotyping further included archived parasites of known DTM susceptibility status. The results identified eleven mtDNA haplotypes, three of which were associated with DTM resistance. Phylogenetic analyses of haplotypes suggested multiple origins of DTM resistance. L. salmonis laboratory strains loA-00 and loA-10 showed similarly high levels ( 100-fold) of DTM resistance in bioassays. Both strains differed strongly in mtDNA haplotype, but shared the missense mutation Leu107Ser in the mitochondrial gene cytochrome c oxidase subunit 1 (COX1), which was detected in all further DTM resistant $L$. salmonis isolates assessed. In crossing experiments with a DTM-susceptible strains, maternal inheritance of DTM resistance is apparent with both loA-10 (this study) and loA-02 (earlier reports). We conclude that Leu107Ser (COX1) is a main genetic determinant of DTM resistance in $L$. salmonis.

\section{Introduction}

Caligid sea lice (Copepoda: Crustacea) are ectoparasite of marine fish, which feed on the mucus, skin and blood of their hosts ${ }^{1}$. In the most severe cases, infestations can lead to skin lesions, secondary infections, osmoregulatory imbalances, induction of endocrine stress responses, reduced appetite and growth, immunosuppression and, if untreated, potentially death ${ }^{2,3}$. Sea lice infections are a major health management problem in the commercial mariculture of Atlantic salmon (Salmo salar L.), with the salmon louse Lepeophtheirus salmonis (Krøyer, 1837) causing most infestation in the North Atlantic. In 2018, the estimated global costs of sea lice infections to the salmon industry were approximately US $\$ 873$ million/ $£ 700$ million ${ }^{4}$, comprising mainly treatment costs and to a lesser extent losses in production.

Sea lice infections of farmed salmon are controlled by integrated pest management strategies combining farm management measures, such as fallowing and the use of single year classes, with a diverse array of non-medicinal control approaches and a limited range of licensed veterinary drugs ${ }^{5,6}$. Non-medicinal sea lice control approaches include alternative cage designs reducing infection pressure ${ }^{7}$, different systems of salmon delousing using physical means such as the application of water jets or brief immersion in warm water ${ }^{8}$, and the co-culture of Atlantic salmon with different species of cleaner fish that remove sea lice from infected salmon ${ }^{9}$. Veterinary drugs available for salmon delousing include the macrocyclic lactone emamectin benzoate and different benzoylureas, which are applied as feed additives, and the organophosphate azamethiphos, the disinfectant hydrogen peroxide, and the pyrethroid deltamethrin (DTM), which are supplied as bath treatments ${ }^{5}$. 
The use of a limited range of salmon delousing agents over more than two decades has led to the evolution of drug resistance in L. salmonis populations of the North Atlantic, with most current veterinary treatments being affected by losses of efficacy 6,10,11. The pyrethroid DTM has been in use as bath treatment against sea lice infections of Atlantic salmon since 1998. While moderate losses of susceptibility were reported in the early $2000 \mathrm{~s}^{12}$, higher levels of resistance have been described more recently ${ }^{13}$. The toxicity of pyrethroids is based on their blocking of arthropod voltagegated sodium channels $\left(\mathrm{Na}_{\mathrm{v}}\right)$, which have essential roles in neurotransmission ${ }^{14}$. Pyrethroid resistance in insects can be based on missense mutations of $\mathrm{Na}_{\mathrm{v}}$ that decrease the channel's affinity to the pesticides ${ }^{15,16}$, or result from the constitutive up-regulation of key enzymes of pesticide detoxification, such as the carboxylesterases (CaEs), cytochrome P450s (CYPs), or glutathione-S-transferases ${ }^{17}$.

In L. salmonis, the molecular mechanism of pyrethroid resistance remains to be resolved. DTM resistance in L. salmonis shows a predominantly maternal mode of inheritance, and is associated with mitochondrial mutations ${ }^{13,18}$. These findings suggest a novel, still unresolved resistance mechanism and imply still unidentified mitochondrial targets for DTM toxicity in susceptible lice. In support of this hypothesis, DTM has been shown to disrupt mitochondrial ATP production ${ }^{13}$ and induce apoptosis in skeletal muscle, which have a high content of mitochondria ${ }^{18}$. Results from crossing experiments suggested additional minor roles of unidentified nuclear genetic factors in DTM susceptibility ${ }^{13}$. In $L$. salmonis, three genes encoding $\mathrm{Na}_{\mathrm{v}}$ channels have been identified ${ }^{19}$, one of which harbours a single nucleotide polymorphism (SNP) that is homologous to a mutation of insect $\mathrm{Na}_{\mathrm{v}}$ decreasing pyrethroid affinity ${ }^{20}$. However, in L. salmonis field populations the $\mathrm{Na}_{\mathrm{v}}$ SNP showed no association with DTM resistance ${ }^{21}$. Similarly, the genomic wide characterization and transcript expression studies of $L$. salmonis CYP and $\mathrm{CaE}$ genes failed to provide evidence for an upregulation of CYPs or CaEs in DTM resistant parasites ${ }^{21,22}$.

In pest or parasite populations, mutations causing pesticide resistance can form de novo or be introduced through migration ${ }^{23}$. Studies determining whether resistance-associated mutations have single or multiple origins can provide insights into the relative importance of mutation as compared to migration in the formation of resistance. Genetic variation between $L$. salmonis populations is weak throughout the North Atlantic, suggesting the species forms a single panmictic population in this geographic area ${ }^{24,25}$. The low degree of genetic differentiation in Atlantic L. salmonis most likely results from the migratory nature of its main host, Atlantic salmon, in which populations reproducing in different freshwater systems share common oceanic feeding grounds during the marine phase of the salmon life cycle $\mathrm{e}^{26}$, providing an opportunity for $L$. salmonis cross-infections. Further contributing to the genetic exchange between $L$. salmonis populations at a more local scale is the parasite's planktonic dispersal at nauplii and copepodid larval stages, which has been estimated to allow for transmission of up to $45 \mathrm{~km}^{27}$.

In accordance with high rates of gene flow between Atlantic L. salmonis, the analysis of haplotypes associated with emamectin benzoate hyposensitivity suggested that resistance against this drug has 
arisen once through de novo mutation followed by a rapid spread throughout the North Atlantic ${ }^{25}$. In contrast, the analysis of SNPs closely linked to a mutation in the L. salmonis ace1a gene, previously shown to confer azamethiphos resistance ${ }^{28}$, revealed multiple and divergent haplotypes associated with resistance ${ }^{29}$. This suggests that the ace1a mutation causing resistance existed prior to the introduction of organophosphate salmon delousing agents, the use of which caused the parallel selection of different haplotypes containing the mutation. In a study of DTM resistance in L. salmonis, four independent highly resistant strain isolates originating from geographically distant Scottish farm sites showed virtually identical mitochondrial genome sequences, contrasting highly polymorphic mitochondrial genome sequences among DTM susceptible samples ${ }^{13}$. While these findings suggest the clonal expansion of DTM-resistance-associated mitochondrial haplotypes as a result of drug selection, the study may have missed rare haplotypes present due to the limited number of individuals analysed.

The present study had the objective of characterising mitochondrial DNA (mtDNA) haplotypes in field populations of $L$. salmonis and assessing their relationship to DTM resistance. L. salmonis were obtained from a range of Scottish farm sites and subjected to bioassays to determine their DTM susceptibility status. Parasites were then genotyped at selected mitochondrial SNP loci to identify mitochondrial haplotypes and assess haplotype association with DTM resistance. Mitochondrial haplotypes were compared to infer whether DTM resistance in salmon lice evolved from single or multiple origins. One resistance-associated haplotype, which differed maximally from a previously studied haplotype, was selected for further investigations. A laboratory L. salmonis strain carrying the haplotype of interest was established and its mitochondrial genome sequenced. The mtDNA of that strain was compared to the mtDNA of previously analysed DTM resistant and susceptible $L$. salmonis strains to derive hypotheses about the potential involvement of mitochondrial SNPs in the resistance mechanisms. The DTM susceptibility of the newly established strain was characterised using drug bioassays and effects of sublethal DTM concentrations on total ATP levels were assessed. The strain was further subjected to crossing experiments to assess the mode of inheritance of DTM resistance.

\section{Materials And Methods}

\section{Ethics statement}

All research projects involving the University of Stirling are subject to a thorough Ethical Review Process prior to any work being approved. The present research was assessed by the University of Stirling Animal Welfare Ethical Review Body (AWERB) and passed the ethical review process. Laboratory infections of Atlantic salmon with $L$. salmonis were performed under a valid UK Home Office license and at low parasite densities unlikely to compromise fish welfare.

\section{Lepeophtheirus salmonis strains and husbandry}

L. salmonis strains were maintained under standardized conditions at the Marine Environmental Research Laboratory of the University of Stirling (Machrihanish, UK), as described in detail elsewhere ${ }^{30}$. 
Strain loA-00 was taken into culture in 2003 and is susceptible to all current salmon delousing agents, while strain loA-02 was established in 2011 and is resistant to emamectin benzoate, DTM, and azamethiphos $^{13,22,31}$. Strain loA-10 was established during this study. To establish this strain, gravid female $L$. salmonis were obtained from an aquaculture production site located on the West coast of Scotland, put into flasks containing cold oxygen-saturated seawater and shipped to the laboratory. Paired egg strings were removed from each parasite and incubated in separate containers as described before to allow hatching and progression to the copepodid stage. The corresponding females were preserved in absolute ethanol and subjected to genotyping assays to establish mitochondrial haplotypes. Based on the results, larvae derived from one dam showing a mitochondrial haplotype of interest were selected and used to infect naïve host fish in order to establish strain loA-10.

This study further included archived $L$. salmonis from nine aquaculture production sites, as well as from wild host fish caught in a salmon river located on the East coast of Scotland, which were stored in absolute ethanol at $-20^{\circ} \mathrm{C}$ pending molecular analyses.

\section{L. salmonis bioassays}

Bioassays were performed to assess the susceptibility of both preadult-II/adult salmon lice and copepodid larvae lice to DTM (PestanaI ${ }^{\circledR}$ analytical standard grade, Sigma-Aldrich, UK), which was dissolved in acetone at a final solvent concentration of $0.05 \%(\mathrm{v} / \mathrm{v})$ in all tests. All bioassay incubations took place in a temperaturecontrolled chamber set to $12^{\circ} \mathrm{C}$.

Bioassays with preadult/adult salmon lice performed within this study had either a standard or a singledose design ${ }^{12,32}$, and have been described in detail elsewhere ${ }^{21}$. In brief, L. salmonis adult males and preadult II/adult females were randomly allocated to $300 \mathrm{~mL}$ crystallising dishes containing $100 \mathrm{~mL}$ of filtered $(55 \mu \mathrm{m})$ seawater, with each dish receiving five females and five males. Standard bioassays included a geometrical series of at least six DTM concentrations in the range of 0.125 and $32 \mu \mathrm{g} / \mathrm{L}$ and a solvent control (Supplementary Table S1) in duplicates. Singledose bioassays were conducted in an analogous fashion, except that DTM was provided at one diagnostic concentration $(2 \mu \mathrm{g} / \mathrm{L})$. Preadult/adult salmon lice were exposed to DTM for $30 \mathrm{~min}$ and then allowed to recover in clean seawater for $24 \mathrm{~h}$ prior to behavioural responses being examined and rated as "live", "weak", "moribund", or "dead". Rating criteria have been described in detail elsewhere ${ }^{31}$.

To generate L. salmonis copepodid larvae for use in bioassay, paired egg strings of gravid females were incubated in aerated filtered seawater in a temperaturecontrolled chamber set to $12^{\circ} \mathrm{C}$, while keeping samples separate. A $1 \mathrm{~mL}$ pipette was used to randomly allocate $1 \mathrm{~mL}$ of filtered seawater with approximately ten copepodids to embryo dishes with glass lids containing $1 \mathrm{~mL}$ of filtered seawater. Chemical exposures were initiated by adding $1 \mu \mathrm{L}$ of a $2000 x$ final concentration solution of DTM to the embryo dishes containing $2 \mathrm{~mL}$ seawater and parasites. Copepodids were exposed to nine concentrations of $\operatorname{DTM}(0.10,0.22,0.46,1.00,2.15,4.64,10.00,21.54$, and $46.42 \mu \mathrm{g} / \mathrm{L})$ and one solvent control, in duplicates. DTM exposure was for $24 \mathrm{~h}$, directly followed by examination and rating of larvae. 
Copepodids were rated "live" when attracted by light and swimming normally, "weak" when swimming irregularly (animals swim in a straight line within 2 min after stimulation using light and a fine brush), "moribund" when incapable of swimming away after stimulation by light and a fine brush (animals may twitch appendages), and "dead" when showing no movements in extremities, gut, or other organs as apparent from examination under a microscope.

Copepodid, preadult, and adult salmon lice rated as "live" or "weak" were considered unaffected, while "moribund" and "dead" parasites were considered affected. In bioassays involving exposure to a series of drug concentrations the susceptibility of the tested population was characterised by probit analysis, while in singledose bioassays involving exposure to $2 \mu \mathrm{g} / \mathrm{L}$ DTM parasites were classified as DTM resistant if rated unaffected and susceptible if rated affected. After completion of bioassays, lice were stored in absolute ethanol at $-20^{\circ} \mathrm{C}$ pending DNA extraction and genetic analyses.

\section{Genotyping of mitochondrial single nucleotide polymorphism (SNP) alleles}

PCR based genotyping assays employing universal fluorescence energy transfer (FRET) probes (KASP® 4.0, LGC Genomics, UK) were designed to detect $18 \mathrm{mtDNA}$ SNPs, including the four nonsynonymous SNPs G8134A, T5889C, T8600C, and G3338A, that have been associated with DTM resistance in $L$. salmonis in a previous study ${ }^{13}$. Genomic DNA was extracted from individual L. salmonis specimens using a high throughput protocol ${ }^{33}$, with details having been reported elsewhere ${ }^{21}$. Each SNP assay involved one common primer and two allele specific primers (Supplementary Table S2), with the method being described in detail elsewhere ${ }^{21}$.

\section{Mitochondrial haplotype network}

A haplotype network was interfered from mitochondrial haplotypes of $L$. salmonis. The network was constructed using the medium-joining method ${ }^{34}$ implemented in the software PopArt v1.7 $7^{35}$. Haplotypes were defined based on the combined occurrence of 18 SNPs that have been associated with DTM resistance in L. salmonis in a previous study ${ }^{13}$.

\section{Mitochondrial genome (mtDNA) amplification and sequencing}

The mitochondrial genome was amplified and sequenced from two salmon lice from the laboratorymaintained strain loA-10. The $L$. salmonis mitochondrial genome was amplified in six overlapping PCRs products using specific oligonucleotide primers that have been designed with Primer3 v4.1.0 (Supplementary Table S3). PCR reactions were performed using $1 \mu \mathrm{l}$ of $50 \mathrm{ng} / \mu \mathrm{L}$ template DNA, $2.5 \mu \mathrm{L}(10 \mathrm{pmol})$ of the forward and the reverse primer, $25 \mu \mathrm{L} \mathrm{Q} 5{ }^{\circledR}$ High-Fidelity $2 \times$ Master Mix (New England BioLabs Ltd, Hitchin, UK), and $19 \mu \mathrm{L}$ nuclease-free water. PCR conditions for each product are listed in Supplementary Supplementary Table S4. All PCR products were purified (NucleoSpin ${ }^{\mathrm{TM}} \mathrm{Gel}$ and PCR Cleanup Kit, Macherey-Nagel, Düren, Germany) and sequenced (Supplementary Table S5) by 
Eurofins Genomics (Ebersberg, Germany). Prior to sequencing, PCR product 6 (Supplementary Table S3) had to be subcloned using a pGEM-T Easy Vector system and chemocompetent Escherichia coli JM-109 (Promega, WI, USA), and plasmids were isolated using a NucleoSpin ${ }^{\mathrm{TM}}$ Plasmid EasyPure kit (MachereyNagel, Düren, Germany). MtDNA sequences obtained for the same individual were manually assessed and trimmed and aligned.

\section{Identification of DTM resistance-associated mtDNA SNP}

MtDNA sequences from loA-10 lice, as well as archived sequences from individuals of strains loA-00 ( $N=$ 2), loA-02 $(N=2)$, loA-01 $(N=4), N A 01-P(N=2)$ and NA01-O $(N=3)$, wild hosts $(N=2)$, and $F 2$ lice from loA-00 and loA-02 crosses $(N=12)^{13}$, were aligned to the Scottish $L$. salmonis mitochondrial reference genome (GenBank: LT630766.1) using the R/Bioconductor package Rbowtie2 v 4.0.3 ${ }^{36}$. Sequence variations were identified using the HaplotypeCallerfunction in GATK v3.5 $5^{37}$. IoA-10 specific SNPs, as well as SNPs common to all DTM resistant individuals and lacking in all susceptible parasites were then identified.

\section{L. salmonis crosses}

The laboratory strains IoA-00, IoA-02, and loA-10 were crossed to produce five families spanning two generations termed parental (P0) and first filial generation (F1). IOA-00, loA-02, and IoA-10 copepodids were maintained on Atlantic salmon smolts in separate tanks to allow development to the adult male and preadultll female stage. Then, half of the fish carrying loA-00 lice and half of the fish carrying loA-10 lice were humanely killed to harvest lice in order to set up two batch crosses. In the first batch cross the P0 generation consisted of IoA-00 adult males and IoA-10 preadultll females, whereas the P0 generation in the second batch cross had the inverse gender-strain orientation. To set up each batch cross, ten Atlantic salmon smolts were anaesthetised using 2-phenoxyethanol (100 mg/L; 99\%; Sigma-Aldrich, U.K.) and received two to three male and female parasites, respectively. The remaining fish carrying loA-00 adult males/preadult-II females and loA-10 adult males/preadultll females, as well as all fish carrying loA-02 adult males/preadult-II females were maintained further, allowing lice to reproduce separately. P0 dams of the five families were maintained to produce egg strings, which were then removed and incubated to allow hatching and larval development ${ }^{38}$. The resulting $\mathrm{F} 1$ copepodid larvae were used to inoculate tanks containing naïve Atlantic salmon. Infections were maintained until F1 parasites reached the adult male and preadult-II female stages. Subsequently, F1 parasites were subjected to bioassays to determine their DTM susceptibility phenotype.

\section{Effects of DTM on L. salmonis ATP levels}

Whole body ATP levels were assessed in L. salmonis from strains IoA-00, IOA-02, and IoA-10 following exposure to sublethal concentrations of DTM and fenpyroximate (Pestanal $\circledast$ analytical standard grade, Sigma-Aldrich, UK). The acaricide fenpyroximate served as a positive control, as it is known to act by interference with the mitochondrial complex $\mathrm{I}^{39}$. 
Drug exposures in ATP experiments followed a similar methodology to that used for bioassays. Adult male salmon lice from strains IoA-00, IOA-02, and IoA-10 were randomly allocated to crystallising dishes containing $100 \mathrm{~mL}$ of filtered seawater, with each dish receiving five adult males. DTM and fenpyroximate were solubilized in acetone $(0.05 \% \mathrm{v} / \mathrm{v}$ in all tests). Parasites were exposed to a solvent control and sublethal concentrations of the tested compounds (DTM: $2 \mu \mathrm{g} / \mathrm{L} ;$ fenpyroximate: $100 \mu \mathrm{g} / \mathrm{L}$ ) in quadruplicate test dishes. Adult males were exposed to DTM for $30 \mathrm{~min}$ and then allowed to recover in clean seawater for $300 \mathrm{~min}$. Exposure to fenpyroximate was for $300 \mathrm{~min}$, without seawater recovery. After drug exposure/seawater recovery, the behavioural response of each animal was rated according to categories given above, and animals deemed alive were added to separate plastic tube containing $1 \mathrm{~mL}$ of Tris-EDTA-saturated phenol (10 mM Tris HCl and $1 \mathrm{mM}$ EDTA; Sigma-Aldrich, UK; Thistle Scientific Ltd, UK). Samples were incubated at room temperature for $10 \mathrm{~min}$ and subsequently stored at $-70^{\circ} \mathrm{C}$. After completion of sampling, frozen samples were submerged in dry ice and transported to the University of Stirling pending ATP analyses. Whole-body ATP levels were measured using a commercially available luciferinluciferase bioluminescence assay kit (A-22066, Molecular Probes, Thermo Fisher Scientific, UK), with details having been reported elsewhere ${ }^{13}$.

\section{Alignment of ND1, ND5, COX1, and COX3 among crustacean species}

Amino acid sequences of ND1, ND5, COX1, and COX3 from L. salmonis and other crustaceans (GenBank accession numbers: Caligus clemensis HQ157566.1, Caligus rogercresseyi HQ157565.1, Tigriopus caligornicus DQ913891.2, Paracyclopina nana EU877959.1, Calanus hyperboreus NC_019627.1, Eucalanus bungii AB091772.1, Squilla mantis NC_006081.1, Tetraclita japonica NC_008974.1, Homarus americanus NC_015607.1, Vargula hilgendorfii NC_005306.1, Daphnia pulex NC_000844.1, Artemia franciscana NC_001620.1, Triops cancriformis NC_004465.1) were aligned using default parameters in the online software Clustal Omega v2.140.

\section{Data analyses and statistical tests}

The dose-response relationship for DTM tested in L. salmonis bioassays was assessed by probit analysis using R package $d r c$ (v3.0-1), assuming a lognormal distribution of drug susceptibility. Based on the fitted models, $\mathrm{EC}_{50}$ and $95 \%$ confidence limits were derived and effects of sex and origin/strain on drug susceptibility assessed.

Whole body ATP levels were expressed as means \pm standard error $(n=15)$. All statistical analyses were performed in R packages car v3.0-11, rcompanion v2.4-1 and PMCMR v4.3. Data were tested for normality and homogeneity of variance using Shapiro-Wilk's and Levene's tests, respectively. As a number of ATP data sets violated these assumptions, the Kruskal-Wallis test was used to assess the effect of drug treatments on ATP levels. The significance level was set at $P<0.05$. The experimentwise type I error was controlled by sequential Bonferroni correction ${ }^{41}$. In those cases where Kruskal-Wallis test results indicated significant differences between treatments, Dunn's test was employed for post-hoc comparisons to the control group. 


\section{Results}

Phenotyping L. salmonis from aquaculture sites, genotyping at four SNP loci

L. salmonis collected at Scottish aquaculture sites were subjected to bioassays involving exposure to 2 $\mu \mathrm{g} / \mathrm{L}$ DTM, with surviving animals failing to show behavioural responses being classified as DTM resistant and animals showing mortality or behavioural responses classified as DTM susceptible. Resistant and susceptible animals from field sites, as well as parasites from previously characterized laboratory strains (IOA-00, DTM susceptible; IoA-02, DTM resistant), were genotyped at four nonsynonymous mtDNA SNP loci, which have previously been shown to be associated with DTM susceptibility ${ }^{13}$. The SNPs used comprised mtDNA mutations G8134A, T5889C, T8600C, and G3338A, which are located in mitochondrial genes NADH dehydrogenase subunit (ND1), NADH dehydrogenase subunit (ND5), cytochrome c oxidase subunit (COX1), and cytochrome c oxidase subunit 3 (COX3), respectively. Based on the combined occurrence of these SNPs, four haplotypes were defined (Table 1). As reported previously, lice of the DTM susceptible strain loA-00 possess haplotype 1, defined by the wild type alleles T8600, G8134, T5889, and G3338, whereas parasites from the DTM resistant strain IoA-02 show haplotype 2, comprising mutant alleles $3338 \mathrm{~A}, 5889 \mathrm{C}, 8134 \mathrm{~A}$, and $8600 \mathrm{C}^{13}$. Genotyping of salmon lice from field populations revealed, in addition to haplotypes 1 and 2, two new haplotypes 3 and 4 . Most animals with haplotype 2,3 and 4 were classified as DTM resistant ( $93 \%, 87 \%$ and $100 \%$, respectively). In contrast, $57 \%$ of individuals with haplotype 1 were rated DTM susceptible, while $43 \%$ were classified resistant (Table 2).

Table 1

Haplotype definitions. Haplotypes 1, 2, 3, and 4 were defined based on the combined occurrence of the four non-synonymous single nucleotide polymorphisms G3338A, T5889C, G8134A, and T8600C, which have been linked to resistance in a previous study ${ }^{13}$.

\begin{tabular}{|llllll|}
\hline Positiona & Alleles & Haplotypes 1 & Haplotypes 2 & Haplotypes 3 & Haplotypes 4 \\
\hline 3338 & G/A & G & A & G & G \\
\hline 5889 & T/C & T & C & T & C \\
\hline 8134 & G/A & G & A & G & A \\
\hline 8600 & T/C & T & C & C & C \\
\hline $\begin{array}{l}\text { a Numbering according to the Scottish L. salmonis mitochondrial reference genome (NCBI Accession } \\
\text { number LT630766.1). }\end{array}$
\end{tabular}


Table 2

Association of mitochondrial haplotypes with deltamethrin resistance. L. salmonis of laboratory strains IoA-00 and IoA-02 and parasites obtained from Scottish aquaculture production sites were subjected to deltamethrin bioassays to establish susceptibility status, followed by genotyping at four SNP loci to establish haplotypes (see Table 1 for details).

\begin{tabular}{|c|c|c|c|c|c|c|}
\hline $\begin{array}{l}\text { Origin of } \\
\text { L. salmonis }\end{array}$ & $\begin{array}{l}\text { Deltamethrin } \\
\text { resistance }^{\mathrm{a}}\end{array}$ & $\mathbf{N}$ & $\begin{array}{l}\text { Haplotype } \\
1\end{array}$ & $\begin{array}{l}\text { Haplotype } \\
2\end{array}$ & $\begin{array}{l}\text { Haplotype } \\
3\end{array}$ & $\begin{array}{l}\text { Haplotype } \\
4\end{array}$ \\
\hline IoA-00 & Susceptible & 22 & 22 & - & - & - \\
\hline IoA-02 & Resistant & 24 & - & 24 & - & - \\
\hline Farm site 1 & Susceptible & 3 & 1 & 2 & - & - \\
\hline (Argyll, 2018) & Resistant & 103 & 3 & 76 & 16 & 8 \\
\hline Farm site 2 & Susceptible & 3 & 1 & 2 & - & - \\
\hline (Argyll, 2018) & Resistant & 51 & 2 & 39 & 4 & 6 \\
\hline Farm site 3 & Susceptible & 7 & - & 4 & 3 & - \\
\hline (Argyll, 2019) & Resistant & $21^{b}$ & - & 16 & 3 & 2 \\
\hline Farm site 4 & Susceptible & 10 & 6 & 3 & 1 & - \\
\hline $\begin{array}{l}\text { (Sutherland, } \\
\text { 2019) }\end{array}$ & Resistant & 20 & 1 & 15 & 4 & - \\
\hline \multirow{3}{*}{$\begin{array}{l}\text { Total (Farm } \\
\text { sites) }\end{array}$} & & 218 & $N=14$ & $N=157$ & $N=31$ & $N=16$ \\
\hline & Susceptible & 23 & $57.1 \%$ & $7 \%$ & $12.9 \%$ & $0 \%$ \\
\hline & Resistant & 195 & $42.9 \%$ & $93 \%$ & $87.1 \%$ & $100 \%$ \\
\hline \multicolumn{7}{|c|}{$\begin{array}{l}\text { a Susceptibility to deltamethrin was determined in single-dose bioassays, involving exposure ( } 30 \mathrm{~min}) \\
\text { to } 2 \mu \mathrm{g} / \mathrm{L} \text { deltamethrin, followed by recovery in seawater ( } 24 \mathrm{~h} \text { ) and subsequent rating as susceptible } \\
\text { (affected) or resistant (unaffected). b } 21 \text { out of a total of } 37 \text { deltamethrin resistant individuals } \\
\text { observed were successfully genotyped. }\end{array}$} \\
\hline
\end{tabular}

\section{Relationship of mtDNA haplotypes to DTM resistance of copepodids}

The relationship between mtDNA haplotypes and DTM resistance was further investigated in $24 \mathrm{~h}$ behavioural bioassays with copepodid larvae derived from gravid females collected at field sites and from laboratory strains. The DTM EC $\mathrm{E}_{50}$ in haplotype 1 copepodids (laboratory strain loA-00) was determined as $0.14 \mu \mathrm{g} / \mathrm{L}$. For copepodid larvae possessing haplotype 2, estimates of the DTM EC $\mathrm{E}_{50}$ value were $4.81 \mu \mathrm{g} / \mathrm{L}$ (laboratory strain loA-02) and $3.98 \mu \mathrm{g} / \mathrm{L}$ (field derived larvae), while copepodids having haplotype 3 showed an $\mathrm{EC}_{50}$ of $6.90 \mu \mathrm{g} / \mathrm{L}$, field derived larvae (Table 3). 
Table 3

Deltamethrin susceptibility and mitochondrial haplotype of $L$. salmonis copepodid larvae. Each bioassay was performed with the copepodid descendants of one female salmon louse. L. salmonis originated from the laboratory strains loA-00 and loA-02, or Scottish aquaculture production sites. Bioassays involved exposure (24 h) to deltamethrin and rating of copepodids as normal or affected. Haplotypes were established based on the genotyping of 10 individuals per bioassay.

\begin{tabular}{|llll|}
\hline $\begin{array}{l}\text { Origin of } \\
\text { L. salmonis }\end{array}$ & $\begin{array}{l}\text { Num } \\
\text { bioassay }\end{array}$ & Haplotype $^{\mathrm{a}}$ & $\begin{array}{l}\mathrm{EC}_{\mathbf{5 0}}(\mathbf{9 5 \%} \mathrm{Cl})^{\mathrm{b}} \\
{[\mu \mathrm{g} / \mathrm{L}]}\end{array}$ \\
\hline IoA-00 & 6 & 1 & $0.14(0.13-0.16)$ \\
\hline IoA-02 & 3 & 2 & $4.81(3.99-5.63)$ \\
\hline Farm site 5, 6 & 2 & 2 & $3.98(2.93-5.04)$ \\
\hline Farm sites 5, 7 & 2 & 3 & $6.90(4.15-9.65)$ \\
\hline $\begin{array}{l}\text { a For definition of haplotypes, see Table 1. }{ }^{\mathrm{b}} \text { Raw data used to derive } \mathrm{EC}_{50} \text { values are provided in } \\
\text { Supplementary Table S10. }\end{array}$ & \\
\hline
\end{tabular}

\section{Further characterization of mitochondrial haplotypes}

In order to further characterise mtDNA diversity in L. salmonis populations of Scottish aquaculture sites, a subset of the above samples from field sites and strains IoA-00 and IOA-02 was further genotyped at 14 mtDNA SNP loci, corresponding to synonymous mutations previously found to be associated with DTM resistance ${ }^{13}$ (Supplementary Table S6). Genotyping further included $L$. salmonis collected from wild hosts in 2010, as well as conserved specimens of the DTM resistant strain NA01-0 ${ }^{13}$. Based on the combined occurrence of the total 18 SNPs considered, the above haplotype definition was refined. Haplotypes 2, 3 and 4 were confirmed by the wider range of SNPs, while haplotype 1 defined on the basis of the initially tested four non-synonymous SNPs was subdivided based on the wider panel of SNPs, differentiating 8 new haplotypes named 1A to $1 \mathrm{H}$ (Supplementary Table S6). Resistanceassociated haplotype 4 was found to be present in specimens removed from wild fish in 2010 and both resistanceassociated haplotypes 3 and 4 were found in individuals of strain NA01-0 established in 2013. A phylogenetic analysis of the 11 haplotypes obtained within this study suggested that haplotype 3 , associated to DTM resistance in bioassays, was closely related to susceptible haplotypes (1A, 1D-F, and $1 \mathrm{H}$ ), but phylogenetically distant to the DTM resistanceassociated haplotypes 2 and 4 (Fig. 1). Further experiments focused on characterising the DTM susceptibility phenotype of $L$. salmonis possessing haplotype 3, which differed from susceptibility-associated haplotypes $1 \mathrm{~A}-\mathrm{H}$ at only one of the 18 tested SNP loci, T8600C in COX1 (Supplementary Table S6).

\section{Establishment and characterization of a laboratory strain with haplotype 3}


The offspring of one field-collected gravid female with haplotype 3 were used to establish a new $L$. salmonis laboratory strain named loA-10. The mitochondrial genome of loA-10 was sequenced (EBI ENA project reference: PRJEB47839), revealing 36 strain-specific sequence variations that were absent in archived sequences from strains IoA-00, IOA-01, loA-02, loA-03, NA01-0, and NA01-P (Supplementary Table S7). The mtDNA genome of IoA-10 strain lice was compared to that of previously analysed DTM resistant, loA-02, IoA-03, NA01-0, NA01-P, and DTM susceptible $L$. salmonis strains, IOA-00, IoA-01 ${ }^{13}$, in order to identify SNPs associated with DTM resistance. The nonsynonymous mtDNA SNP T8600C, corresponding to Leu107Ser in COX1, and eight synonymous SNPs were the only mutations shared by all resistant and lacking in all susceptible parasites (Table 4).

Table 4

Mitochondrial sequence variations specific for deltamethrin resistant $L$. salmonis isolates. The mitochondrial genome (mtDNA) was compared between deltamethrin resistant (IoA-02, loA-03, IoA-10, NA01-O, NA01-P) and susceptible (IOA-00, loA-01) L. salmonis strains to establish mutations associated with resistance. The mtDNA sequence of strain loA-10 was established by PCR followed by Sanger sequencing within the present study, while that of the other strains has been reported previously ${ }^{13}$.

\begin{tabular}{|llllll|}
\hline Position $^{\text {a }}$ & Type & Location & Description & Reference & Alternative \\
\hline 812 & Polymorphism & D-loop & T & C \\
\hline 875 & Polymorphism & D-loop & T & C \\
940 & Deletion & D-loop & AG & A \\
\hline 960 & Polymorphism & D-loop & T & C \\
963 & Polymorphism & D-loop & G & A \\
972 & Polymorphism & D-loop & A & G \\
\hline 8600 & $\begin{array}{l}\text { Non-synonymous } \\
\text { mutation }\end{array}$ & COX1 & TTG/Leu & T & C \\
\hline 10178 & Polymorphism & I-rRNA & A & G \\
\hline 15377 & Polymorphism & D-loop & G & A \\
\hline $\begin{array}{l}\text { a Numbering according to the Scottish } \text { L. salmonis mitochondrial reference genome (NCBI Accession } \\
\text { number LT630766.1). }\end{array}$ & & & \\
\hline
\end{tabular}

To assess the mode by which DTM resistance of strain loA-10 is passed on to the next generation, reciprocal crosses were performed between this strain and the drug-susceptible strain loA-00 to produce two sets of F1 (filial generation 1) lice (Supplementary Table S9). The DTM susceptibility of parental and F1 populations was determined in bioassays (Supplementary Table S11). Strains loA-02 and IoA-10, as well as $\mathrm{F} 1$ offspring derived from loA-10 dams and loA-00 sires, were highly DTM resistant $\left(\mathrm{EC}_{50}\right.$ values > $24.0 \mu \mathrm{g} / \mathrm{L}$; Fig. 2). In contrast, F1 animals derived from loA-00 dams and loA-10 sires ( $E_{50} 0.55 \mu \mathrm{g} / \mathrm{L}$; Fig. 2) were only marginally but significantly $(P<0.0001)$ less susceptible to DTM than loA-00 lice $\left(E_{50}\right.$ $0.25 \mu \mathrm{g} / \mathrm{L}$; Fig. 2). 
To further characterise DTM toxicity in loA-10 lice, the effects of DTM exposure on wholebody ATP levels in strain loA-10 were investigated. Experiments further included fenpyroximate $(100 \mu \mathrm{g} / \mathrm{L})$, an acaricide known to block oxidative phosphorylation. As expected, fenpyroximate caused toxicity, and decreased whole-body ATP levels in all strains assessed (Supplementary Table S10 \& Fig. 3). Exposure to $2 \mu \mathrm{g} / \mathrm{L}$ DTM caused toxic effects accompanied by significantly decrease of ATP levels in loA-00 lice $(P=0.008)$, but failed at causing toxicity or significant effects on the ATP levels in loA-02 and loA-10 lice.

\section{Protein sequence comparisons of ND1, ND5, COX1, and COX3 among crustacean species}

Amino acid sequences of ND1, ND5, COX1, and COX3 from L. salmonis and different crustacean species for which mtDNA sequences were available were aligned to reveal conserved residues within these mitochondrial polypeptides. Among four nonsynonymous mtDNA SNP loci that have previously been reported from a DTM resistant strain ${ }^{13}$, G3338A (COX3 Gly33Glu), T5889C (ND5 Leu411Ser) and G8134A (ND1 Gly251Ser) cause residue changes at non-conserved positions, while T8600C (COX1 Leu107Ser) alters the amino acid sequence at a position that is conserved among all species assessed (Supplementary Fig. S1).

\section{Discussion}

Previous studies have shown that DTM resistance in L. salmonis is transmitted predominantly by maternal inheritance and associated with mtDNA SNPs ${ }^{13,42}$. While an earlier study suggested that highly DTM resistant $L$. salmonis share virtually identical mtDNA sequences ${ }^{13}$, the present study identified three mtDNA haplotypes associated with resistance, one of which coincides with the previously reported resistance associated mtDNA sequence. $L$. salmonis strain loA-10, which possesses one of the novel DTM resistance associated haplotypes, showed a high level of DTM resistance comparable to that of the previously characterised strain loA02 and, similar to loA02, passed on DTM resistance to the next generation through maternal inheritance. The phylogenetic analysis of mtDNA haplotypes provided evidence for multiple origins of mtDNAassociated DTM resistance in L. salmonis. Comparison of mtDNA sequences between DTM resistant and susceptible salmon louse strains suggested the association of DTM resistance with SNP T8600C, corresponding to Leu107Ser in COX1.

L. salmonis obtained at aquaculture sites were subjected to bioassays to determine their DTM susceptibility status. Parasites were then genotyped at previously described mtDNA SNP $10 \mathrm{i}^{13}$ to identify mitochondrial haplotypes and assess haplotype association with DTM resistance. 93\%, 87\%, and 100\% of lice possessing haplotypes 2, 3, and 4, respectively, were classified as DTM resistant, emphasising the association of mtDNA mutations with the resistance phenotype. The remaining 7-13\% may have died due to interacting environmental factors or handling of parasites during sampling, transportation, and set-up of bioassays rather than drug toxicity ${ }^{10,43}$. As bioassays were performed on lice directly obtained from farmed salmon, environmental conditions prior sampling could not be controlled. Lice may have also 
been exposed to stressful conditions during sampling and due to abrupt changes of environmental conditions during transportation. The DTM resistance phenotype was less distinct for lice containing haplotype 1 . While most lice with this haplotype were classified as DTM susceptible, $43 \%$ were rated resistant. The large number of resistant haplotype 1 individuals suggests the contribution of genetic factors other than mitochondrial mutations to the DTM resistance phenotype, which is in line with findings by Carmona-Antoñanzas et al. ${ }^{13}$ who attributed the presence of about $20 \%$ resistant $\mathrm{F} 2$ parasites in a family descending from a DTM susceptible dam and a resistant sire to nuclear genetic determinants of resistance.

To address confounding factors in bioassays on lice directly collected from farmed salmon, this study included analogous experiments with copepodid larvae derived from eggs of one female. When investigating effects of mtDNA mutations on drug resistance, advantages of F1 bioassays with copepodid larvae are that sibling clutches share the same mitochondrial haplotype ${ }^{44}$ and were hatched and reared under standardized laboratory conditions less likely to bias the outcome of the experiment. While insufficient gravid females of appropriate genotype precluded analysis of haplotype 4 in copepodid bioassays, testing of larvae of the remaining haplotypes resolved significant differences in drug resistance between $L$. salmonis of haplotypes 2 and 3 as compared to parasites of haplotype 1 , confirming results from conventional bioassays and underpinning the association of mtDNA mutations with DTM resistance. Moreover, the study showed that DTM resistance is already present in the larval stage, which would be expected for resistance conferred by mtDNA mutations ${ }^{13}$.

Genotyping of field isolates collected in 2018 and 2019 revealed three DTM resistance-associated mtDNA haplotypes, with haplotypes 2,3 , and 4 being found in $75 \%, 14 \%$ and $8 \%$ of all resistant lice, respectively. Analysis of archived samples further provided evidence for haplotypes 4 being present in parasites removed from wild salmon in 2010, while haplotypes 3 and 4 were found in the DTM resistant strain NA01-0, which was established in 2013. These findings contrast with results from Carmona-Antoñanzas et al. ${ }^{13}$, who reported mtDNA sequences consistent with haplotype 2 for all DTM resistant isolates analysed in their study. Moreover, no resistance-associated haplotypes were evident from parasites collected from wild salmon in 2010. The failure to detect the less frequent haplotypes 3 and 4 in the earlier study ${ }^{13}$ could be attributed to the limited number of sequenced individuals.

Phylogenetic analyses of mtDNA haplotypes showed that the three DTM resistance-associated haplotypes fall into two clusters, with haplotype 3 being phylogenetically distant to haplotypes 2 and 4 , which shared 17 out of 18 tested SNP loci. This mtDNA sequence variability in DTM resistant isolates indicate that mtDNA-associated DTM resistance in L. salmonis originated from at least two independent origins. Thus, resistanceassociated mitochondrial mutation(s) may have been selected for, more or less in parallel, as a consequence of the extensive use of pyrethroids.

Resistance-associated haplotypes 2 and 3 differed maximally in sequence, with only one out of 18 tested SNP loci being shared. To investigate the relationship of haplotypes 2 and 3 to DTM resistance, the present study performed comparative experiments with strain loA-02 containing haplotype 2 and strain 
loA-10 containing haplotype 3. Despite haplotypes 2 and 3 being very different in sequence, their resistance phenotype is very similar. Both loA-10 (haplotype 3 ) and loA-02 (haplotype 2) lice were highly DTM resistant $\left(E_{50}\right.$ values $\left.>24.0 \mu \mathrm{g} / \mathrm{L} ; \mathrm{P}=0.845\right)$ and reciprocal crosses of strains loA-10 (present study) and IoA-0 $2^{13}$ with the drugsusceptible loA00 lice revealed that both strains transmit their resistance to the next generation through maternal inheritance. Moreover, DTM exposure caused behavioural toxicity and wholebody ATP depletion in DTM susceptible loA-00 parasites, but not resistant IoA-10 and loA-02 lice. These findings are in line with an earlier experiment, which compared the effect of DTM exposure on behavioural toxicity and ATP levels between loA-00 and loA-02 lice ${ }^{13}$. The maternal inheritance of DTM resistance in families derived from a resistant dam provide evidence that the resistance phenotype is conferred by the maternally transmitted mitochondrial genome, which has been discussed in detail elsewhere ${ }^{13}$. Depletion of ATP levels in DTM susceptible lice may be related to the toxic effect of DTM on the mitochondria, and mtDNA mutations in haplotypes 2 and 3 may have a protective effect.

As the inheritance of mtDNA is linear and lacks recombination through meiosis, relevant SNPs for DTM resistance are transmitted together with irrelevant hitchhiking SNPs ${ }^{44}$. Thus, SNPs that are truly linked to DTM resistance are expected to be present in all resistanceassociated haplotypes but lacking in all susceptibilityassociated haplotypes. The nonsynonymous mtDNA SNP T8600C, corresponding to Leu107Ser in COX1, was the only mutation shared by the resistance-associated haplotypes 2, 3, and 4 and lacking in all susceptibility-associated haplotypes. When comparing mtDNA sequences of DTM resistant and susceptible lice, T8600C was also the only non-synonymous mutation differentiating between resistant and susceptible individuals. Sequencing analyses further revealed eight additional SNPs in non-coding regions (NCR) of the mtDNA that were common to all resistant lice and lacking in all susceptible lice. NCR sequences are the most variable mtDNA sequences, which may explain the high number of SNPs found within this region in the present study ${ }^{45}$. Seven of these SNPs were found in the mitochondrial control region, also known as displacement loop (D-loop). Its function is not yet fully understood but seems to be critical in regulating replication and transcription of mtDNA ${ }^{46}$. However, Dloop mutations are not known to confer drug resistance. Another SNP, A10178G, was found within a mitochondrial ribosomal RNA (rRNA) gene and has also been described by Bakke et al. ${ }^{18}$. Mitochondrial rRNAs are assembled with ribosomal proteins encoded by nuclear genes to form mitochondrial ribosomes, which are responsible for translating mitochondrial proteins ${ }^{47}$. Thus, mutation within the mitochondrial rRNA may lead to ribosome dysfunction and may result in respiratory chain defects ${ }^{48}$. However, to our knowledge, there are no reports of mitochondrial rRNA mutations associated with drug resistance.

Findings of the present study raise questions about the mechanism of DTM resistance and by inference the mechanism of DTM toxicity in L. salmonis. While it is generally accepted that pyrethroids target $\mathrm{Na}_{\mathrm{v}}$ in terrestrial arthopods ${ }^{14}$, several studies with terrestrial arthropods and mammals provide evidence for pyrethroid effects on mitochondrial functions. Due to their lipophilic nature, pyrethroids can pass and interact with biological membranes, making mitochondrial membranes and membrane proteins 
candidate targets for toxic action ${ }^{49}$. For example, pyrethroids have been shown to affect mitochondrial membrane structures and dynamics, which can impair oxidative phosphorylation ${ }^{50,51}$. Mitochondrial oxidative phosphorylation can also be impaired by intracellular Ca2+ accumulation ${ }^{52,53}$, which can result from interactions of pyrethroids with Nav and consequent $\mathrm{Ca} 2+$ influx ${ }^{54}$ and direct effects of pyrethroids on voltage-gated $\mathrm{Ca} 2+$ channels $^{55}$.

Pyrethroid induced disruption of mitochondrial membrane integrity and inhibition of respiratory complexes, as well as intracellular $\mathrm{Ca} 2+$ accumulation can cause the generation of reactive oxygen species (ROS) in mitochondria ${ }^{56-58}$. ROS can trigger a cascade of reactions that induces lipid peroxidation and damage of macromolecules ${ }^{59-61}$. Pyrethroids have been shown to induce intrinsic mitochondrial apoptosis $^{62,63}$, which involves mitochondrial outer membrane permeabilization, release of cytochrome $C$ into the cytosol, activation of caspases, and ultimately DNA fragmentation ${ }^{64}$. In particular, this pathway can be triggered by pyrethroid induced oxidative stress and $\mathrm{Ca}^{2+}$ accumulation, as well as

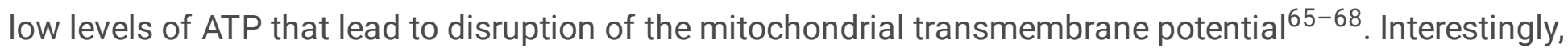
DTM exposure increased apoptosis in mitochondria-rich skeletal muscle, subcuticular tissue, and central ganglion cells in salmon lice of a drug susceptible strain, but not or to a lesser degree in a DTM resistant strain $^{18}$.

Taken together, in salmon lice, DTM may induce toxicity through disruption of mitochondrial membranes, direct inhibition of mitochondrial respiratory complex(es), intracellular $\mathrm{Ca}^{2+}$ accumulation, or by causing oxidative stress. An obvious explanation for DTM toxicity in salmon lice would be that DTM or its metabolites are binding to a mitochondrial respiratory complex and lead to disruption of the mitochondrial ATP production, which has been observed in the present study. In addition, inhibition of respiratory complexes can lead to the formation of ROS ${ }^{56}$. Both, low levels of ATP and oxidative stress can in turn induce intrinsic mitochondrial apoptosis ${ }^{65,66,68}$, which has been described by Bakke et al. ${ }^{18}$. Resistance may be conferred by mitochondrial SNP(s) that changes the amino-acid sequences of the complex and impair binding of DTM. For reasons explained above, T8600C leading to Leu107Ser in COX1 is the most probable mutation for conferring DTM resistance in salmon lice. Alternatively, DTM might impair the mitochondrial ATP production in susceptible lice by causing disruptions of the mitochondrial membrane or by secondary effects arising from DTM toxicity. In these scenarios, mtDNA mutation(s) may have functional effects on the efficiency of electron transfer or proton translocation, counteracting ATP deficits.

\section{Conclusion}

DTM resistance in L. salmonis is associated with multiple mtDNA haplotypes which have multiple origins. Non-synonymous mtDNA mutation T8600C, corresponding to Leu107Ser in COX1, was common to all DTM resistant mtDNA haplotypes but lacking in haplotypes not associated with resistance. Parasites possessing a mtDNA haplotype in which T8600C was the only non-synonymous mutation are 
highly DTM resistant and pass on their resistance to the next generation through maternal inheritance. The results suggest the association of DTM resistance with SNP T8600C (Leu107Ser in COX1).

\section{Declarations}

\section{Acknowledgments}

This work was supported by a PhD studentship awarded to C.T. by PHARMAQ/Zoetis. This study was further supported by United Kingdom Biotechnology and Biological Sciences Research Council grant BB/L022923/1 awarded to A.S. The authors further gratefully acknowledge funding received from the MASTS pooling initiative (The Marine Alliance for Science and Technology for Scotland). MASTS is funded by the Scottish Funding Council (grant reference HR09011) and contributing institutions. The funders had no role in study design, data analysis or interpretation of results. For the purpose of open access, the author has applied a CC BY public copyright licence to any Author Accepted Manuscript version arising from this submission.

\section{Author contributions}

All authors conceived the experiment, C.T. conducted the experiment, C.T., A.S and M.B. analysed the data. C.T., A.S., M.B. and J.E.B. reviewed and discussed the results. All authors reviewed the manuscript.

\section{Competing interests}

The authors declare no competing interests.

\section{Data availability}

The sequencing data were submitted to the EBI ENA database under the Project number PRJEB47839.

\section{References}

1. Boxaspen, K. A review of the biology and genetics of sea lice. ICES J. Mar. SCi, 63, 1304-1316 (2006).

2. Grimnes, A. \& Jakobsen, P. J. The physiological effects of salmon lice infection on post-smolt of Atlantic salmon. J. Fish Biol, 48, 1179-1194 (1996).

3. Wootten, R., Smith, J. W. \& Needham, E. A. Aspects of the biology of the parasitic copepods Lepeophtheirus salmonis and Caligus elongatus on farmed salmonids, and their treatment. Proc. R. Soc. Edinburgh. Sect. B. Biol. Sci. 81, 185-197(1982). 
4. Brooker, A. J., Skern-Mauritzen, R. \& Bron, J. E. Production, mortality, and infectivity of planktonic larval sea lice, Lepeophtheirus salmonis (Krøyer, 1837): current knowledge and implications for epidemiological modelling. ICES J. Mar. Sci, 75, 1214-1234 (2018).

5. Burridge, L., Weis, J. S., Cabello, F., Pizarro, J. \& Bostick, K. Chemical use in salmon aquaculture: A review of current practices and possible environmental effects., 306, 7-23 (2010).

6. Torrissen, 0. et al. Salmon lice - impact on wild salmonids and salmon aquaculture. J. Fish Dis, 36, 171-194 (2013).

7. Stien, L. H. et al. 'Snorkel' sea lice barrier technology reduces sea lice loads on harvest-sized Atlantic salmon with minimal welfare impacts., 458, 29-37 (2016).

8. Overton, K. et al. Salmon lice treatments and salmon mortality in Norwegian aquaculture: a review. Rev. Aquac, 11, 1398-1417 (2019).

9. Brooker, A. J. et al. Sustainable production and use of cleaner fish for the biological control of sea lice: recent advances and current challenges. Vet. Rec, 183, 383-383 (2018).

10. Aaen, S. M., Helgesen, K. O., Bakke, M. J., Kaur, K. \& Horsberg, T. E. Drug resistance in sea lice: a threat to salmonid aquaculture. Trends Parasitol, 31, 72-81 (2015).

11. Myhre Jensen, E., Horsberg, T. E., Sevatdal, S. \& Helgesen, K. O. Trends in de-lousing of Norwegian farmed salmon from 2000-2019-Consumption of medicines, salmon louse resistance and nonmedicinal control methods. PLoS One, 15, e0240894 (2020).

12. Sevatdal, S. \& Horsberg, T. E. Determination of reduced sensitivity in sea lice (Lepeophtheirus salmonis Krøyer) against the pyrethroid deltamethrin using bioassays and probit modelling., 218, 21-31 (2003).

13. Carmona-Antoñanzas, G. et al. Maternal inheritance of deltamethrin resistance in the salmon louse Lepeophtheirus salmonis (Krøyer) is associated with unique mtDNA haplotypes. PLoS One, 12, e0180625 (2017).

14. Davies, T. G. E. E., Field, L. M., Usherwood, P. N. R. R. \& Williamson, M. S. DDT, pyrethrins, pyrethroids and insect sodium channels. IUBMB Life, 59, 151-162 (2007).

15. Knipple, D. C., Doyle, K. E., Marsella-Herrick, P. A. \& Soderlund, D. M. Tight genetic linkage between the $\mathrm{kdr}$ insecticide resistance trait and a voltage-sensitive sodium channel gene in the house fly. Proc. Natl. Acad. Sci. 91, 2483-2487(1994).

16. Williamson, M. S., Denholm, I., Bell, C. A. \& Devonshire, A. L. Knockdown resistance (kdr) to DDT and pyrethroid insecticides maps to a sodium channel gene locus in the housefly (Musca domestica). Mol. Gen. Genet, 240, 17-22 (1993).

17. Ranson, H. et al. Evolution of supergene families associated with insecticide resistance. Science, 298, 179-181 (2002).

18. Bakke, M. J., Agusti, C., Bruusgaard, J. C., Sundaram, A. Y. M. \& Horsberg, T. E. Deltamethrin resistance in the salmon louse, Lepeophtheirus salmonis (Krøyer): Maternal inheritance and reduced apoptosis. Sci. Rep, 8, 8450 (2018). 
19. Carmona-Antoñanzas, G. et al. Mutations in voltage-gated sodium channels from pyrethroid resistant salmon lice (Lepeophtheirus salmonis). Pest Manag. Sci, 75, 527-536 (2019).

20. Usherwood, P. N. R. et al. Mutations in DIIS5 and the DIIS4-S5 linker of Drosophila melanogaster sodium channel define binding domains for pyrethroids and DDT. FEBS Lett, 581, 5485-5492 (2007).

21. Tschesche, C. et al. Investigation of deltamethrin resistance in salmon lice (Lepeophtheirus salmonis) provides no evidence for roles of mutations in voltage-gated sodium channels. Pest Manag. Sci, 77, 1052-1060 (2021).

22. Humble, J. L. et al. Genome-wide survey of cytochrome P450 genes in the salmon louse Lepeophtheirus salmonis (Krøyer, 1837). Parasit. Vectors, 12, 563 (2019).

23. ffrench-Constant, R. H., Daborn, P. J. \& Goff, G. Le. The genetics and genomics of insecticide resistance. Trends Genet, 20, 163-170 (2004).

24. Glover, K. et al. Population genetic structure of the parasitic copepod Lepeophtheirus salmonis throughout the Atlantic. Mar. Ecol. Prog. Ser, 427, 161-172 (2011).

25. Besnier, F. et al. Human-induced evolution caught in action: SNP-array reveals rapid amphi-atlantic spread of pesticide resistance in the salmon ecotoparasite Lepeophtheirus salmonis. BMC Genomics, 15, 937 (2014).

26. Jacobsen, J. A. et al. Distribution by origin and sea age of Atlantic salmon (Salmo salar) in the sea around the Faroe Islands based on analysis of historical tag recoveries. ICES J. Mar. Sci, 69, 15981608 (2012).

27. Johnsen, I., Asplin, L., Sandvik, A. \& Serra-Llinares, R. Salmon lice dispersion in a northern Norwegian fjord system and the impact of vertical movements. Aquac. Environ. Interact, 8, 99-116 (2016).

28. Kaur, K., Helgesen, K. O., Bakke, M. J. \& Horsberg, T. E. Mechanism behind resistance against the organophosphate azamethiphos in salmon lice (Lepeophtheirus salmonis). PLoS One, 10, e0124220 (2015).

29. Kaur, K. et al. The mechanism (Phe362Tyr mutation) behind resistance in Lepeophtheirus salmonis pre-dates organophosphate use in salmon farming. Sci. Rep, 7, 12349 (2017).

30. Heumann, J., Carmichael, S., Bron, J. E., Tildesley, A. \& Sturm, A. Molecular cloning and characterisation of a novel P-glycoprotein in the salmon louse Lepeophtheirus salmonis. Comp. Biochem. Physiol. Part C Toxicol. Pharmacol, 155, 198-205 (2012).

31. Carmona-Antoñanzas, G. et al. Time-to-response toxicity analysis as a method for drug susceptibility assessment in salmon lice., 464, 570-575 (2016).

32. Helgesen, K. O. \& Horsberg, T. E. Single-dose field bioassay for sensitivity testing in sea lice, Lepeophtheirus salmonis: development of a rapid diagnostic tool. J. Fish Dis, 36, 261-272 (2013).

33. Montero-Pau, J., Gómez, A. \& Muñoz, J. Application of an inexpensive and high-throughput genomic DNA extraction method for the molecular ecology of zooplanktonic diapausing eggs. Limnol. Oceanogr. Methods, 6, 218-222 (2008). 
34. Bandelt, H. J., Forster, P. \& Rohl, A. Median-joining networks for inferring intraspecific phylogenies. Mol. Biol. Evol, 16, 37-48 (1999).

35. Leigh, J. W. \& Bryant, D. POPART: full-feature software for haplotype network construction. Methods Ecol. Evol, 6, 1110-1116 (2015).

36. Wei, Z., Zhang, W., Fang, H., Li, Y. \& Wang, X. esATAC: an easy-to-use systematic pipeline for ATACseq data analysis., 34, 2664-2665 (2018).

37. McKenna, A. et al. The Genome Analysis Toolkit: A MapReduce framework for analyzing nextgeneration DNA sequencing data. Genome Res, 20, 1297-1303 (2010).

38. Heumann, J., Carmichael, S. N., Bron, J. E. \& Sturm, A. Isolation and characterisation of four partial CDNA sequences encoding multidrug resistance-associated proteins (MRPs) in the salmon louse Lepeophtheirus salmonis (Krøyer, 1837)., 424-425, 207-214 (2014).

39. Lümmen, P. Complex I inhibitors as insecticides and acaricides. Biochim. Biophys. Acta, 1364, 287296 (1998).

40. Sievers, F. \& Higgins, D. G. Clustal Omega for making accurate alignments of many protein sequences. Protein Sci, 27, 135-145 (2018).

41. Sugawara, E. \& Nikaido, H. Properties of AdeABC and AdelJK efflux systems of Acinetobacter baumannii compared with those of the AcrAB-TolC system of Escherichia coli. Antimicrob. Agents Chemother, 58, 7250-7257 (2014).

42. Nilsen, F. \& Espedal, P. G. Method for detection of pyrethroid resistance in crustaceans and oligonucleotide sequences useful in detection of pyrethroid resistance.European Patent OfficeEP3030674A1 (2014).

43. Robertson et al. Bioassays with Arthropods (CRC Press, 2017). doi:10.1201/9781315373775.

44. Scheffler, I. E. A century of mitochondrial research: achievements and perspectives., 1, 3-31 (2001).

45. Nicholls, T. J. \& Minczuk, M. In D-loop: 40 years of mitochondrial 7S DNA. Exp. Gerontol, 56, 175-181 (2014).

46. Ciesielski, G. L., Oliveira, M. T. \& Kaguni, L. S. Animal mitochondrial DNA replication. Enzym, 39, 255292 (2016).

47. Sylvester, J. E., Fischel-Ghodsian, N. \& Mougey, E. B. \& O'brien, T. W. Mitochondrial ribosomal proteins: Candidate genes for mitochondrial disease. Genet. Med, 6, 73-80 (2004).

48. Smith, P. M. et al. The role of the mitochondrial ribosome in human disease: searching for mutations in 12S mitochondrial rRNA with high disruptive potential. Hum. Mol. Genet, 23, 949-967 (2014).

49. Guven, C., Sevgiler, Y. \& Taskin, E. Pyrethroid insecticides as the mitochondrial dysfunction inducers. in Mitochondrial Diseases (eds. Taskin, E., Guven, C. \& Sevgiler, Y.) 293-322(IntechOpen, 2018). doi:10.5772/intechopen.80283.

50. Zhang, S. Y. et al. Permethrin may disrupt testosterone biosynthesis via mitochondrial membrane damage of Leydig cells in adult male mouse., 148, 3941-3949 (2007). 
51. Braguini, W. L., Cadena, S. M. S. C., Carnieri, E. G. S., Rocha, M. E. M. \& de Oliveira, M. B. M. Effects of deltamethrin on functions of rat liver mitochondria and on native and synthetic model membranes. Toxicol. Lett, 152, 191-202 (2004).

52. Paschen, W. Role of calcium in neuronal cell injury: which subcellular compartment is involved? Brain Res. Bull, 53, 409-413 (2000).

53. Bauer, T. M. \& Murphy, E. Role of mitochondrial calcium and the permeability transition pore in regulating cell death. Circ. Res, 126, 280-293 (2020).

54. Hossain, M. M. \& Richardson, J. R. Mechanism of pyrethroid pesticide-induced apoptosis: role of calpain and the ER stress pathway. Toxicol. Sci. an Off. J. Soc. Toxicol, 122, 512-525 (2011).

55. Clark, J. M. \& Symington, S. B. Advances in the mode of action of pyrethroids. Topics in current chemistry, 314, 49-72 (2011).

56. Sipos, I., Tretter, L. \& Adam-Vizi, V. The production of reactive oxygen species in intact isolated nerve terminals is independent of the mitochondrial membrane potential. Neurochem. Res, 28, 1575-1581 (2003).

57. Truong, D. H., Eghbal, M. A., Hindmarsh, W., Roth, S. H. \& O’Brien, P. J. Molecular mechanisms of hydrogen sulfide toxicity. Drug Metab. Rev, 38, 733-744 (2006).

58. Brookes, P. S., Yoon, Y., Robotham, J. L., Anders, M. W. \& Sheu, S. S. Calcium, ATP, and ROS: a mitochondrial love-hate triangle. Am. J. Physiol. Cell Physiol, 287, C817-33 (2004).

59. Fang, Y. Z., Yang, S. \& Wu, G. Free radicals, antioxidants, and nutrition. Nutrition, 18, 872-879 (2002).

60. Chirico, S., Smith, C., Marchant, C., Mitchinson, M. J. \& Halliwell, B. Lipid peroxidation in hyperlipidaemic patients. A study of plasma using an HPLC-based thiobarbituric acid test. Free Radic. Res. Commun, 19, 51-57 (1993).

61. Maiti, P. K., Kar, A., Gupta, P. \& Chaurasia, S. S. Loss of membrane integrity and inhibition of type-I iodothyronine 5'-monodeiodinase activity by fenvalerate in female mouse. Biochem. Biophys. Res. Commun, 214, 905-909 (1995).

62. Kumar, A. et al. Deltamethrin-induced oxidative stress and mitochondrial caspase-dependent signaling pathways in murine splenocytes. Environ. Toxicol, 31, 808-819 (2016).

63. Ko, J., Park, J. H., Park, Y. S. \& Koh, H. C. PPAR-y activation attenuates deltamethrin-induced apoptosis by regulating cytosolic PINK1 and inhibiting mitochondrial dysfunction. Toxicol. Lett, 260, 8-17 (2016).

64. Bock, F. J. \& Tait, S. W. G. Mitochondria as multifaceted regulators of cell death. Nat. Rev. Mol. Cell Biol, 21, 85-100 (2020).

65. Buttke, T. M. \& Sandstrom, P. A. Oxidative stress as a mediator of apoptosis. Immunol. Today, 15, 710 (1994).

66. Lenaz, G. et al. Role of mitochondria in oxidative stress and aging. Ann. N. Y. Acad. Sci, 959, 199213 (2002). 
67. Hajnóczky, G., Davies, E. \& Madesh, M. Calcium signaling and apoptosis. Biochem. Biophys. Res. Commun, 304, 445-454 (2003).

68. Kroemer, G. Mitochondrial control of apoptosis: an introduction. Biochem. Biophys. Res. Commun, 304, 433-435 (2003).

\section{Figures}
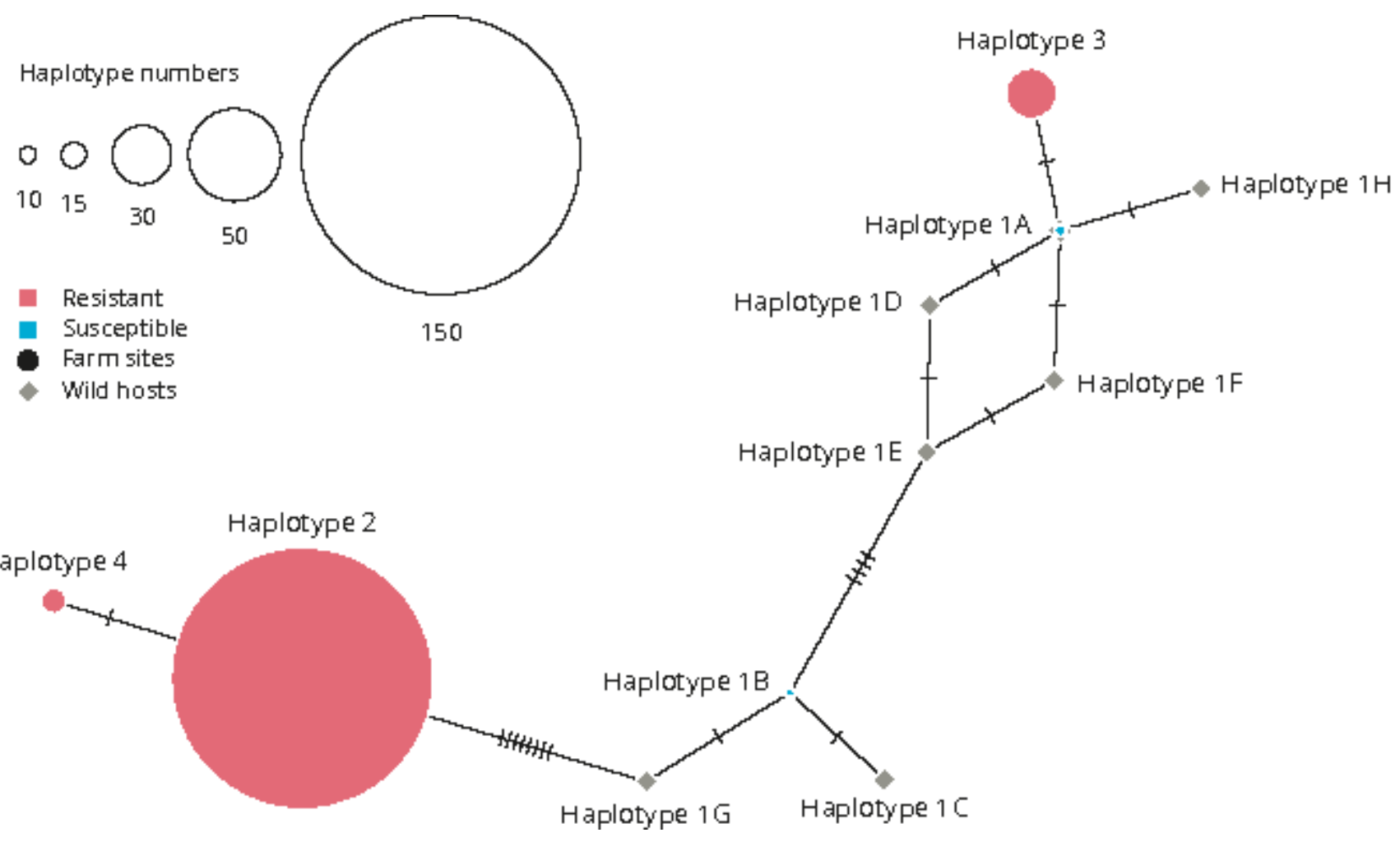

\section{Figure 1}

Median-joining haplotype network interfered from mitochondrial haplotypes of L. salmonis. Haplotypes were defined based on the combined occurrence of 18 mitochondrial SNPs, which have been associated with deltamethrin resistance in L. salmonis in a previous study 13 (Supplementary Tables S6 and S7). Haplotypes that were identified in salmon lice from farm sites are represented by circles, while haplotypes that were identified in salmon lice from wild hosts are represented by grey rhombi. The size of each circle is proportional to the frequency of each haplotype in salmon lice from farm sites. Deltamethrin resistance associated haplotypes are represented by red circles and susceptibility-associated haplotypes are shown in blue. 

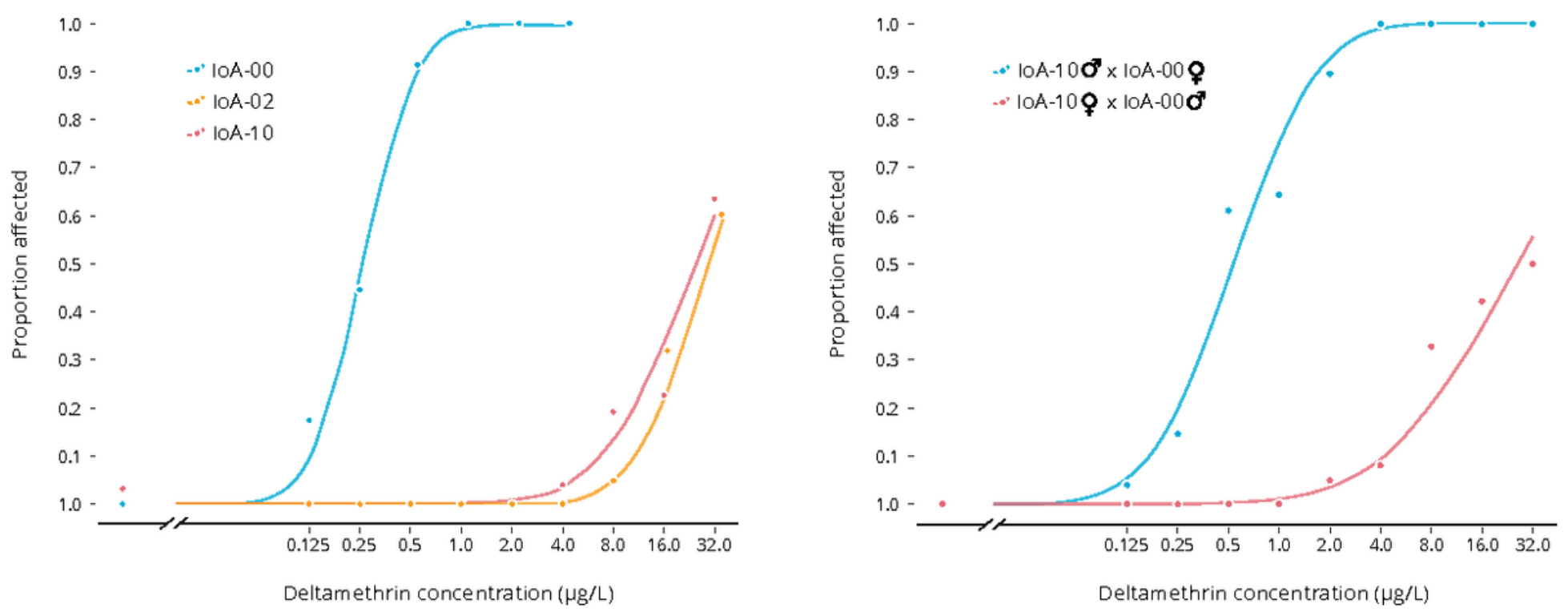

Figure 2

Standard deltamethrin bioassay with L. salmonis first filial (F1) progenies derived from parental (P0) crosses of different gender-strain orientations. Median effective concentrations EC50 [ $\mathrm{\mu g} / \mathrm{L}]$ and $95 \%$ confidence limits: IoA-10 = 24.73 (15.06-34.39), loA-00 = 0.25 (0.20-0.30), loA-02 = 25.95 (17.98-33.92), F1: IoA-10 dam x loA-00 sire = 26.10 (11.46-40.75), F1: IoA 10 sire $x$ loA-00 dam = 0.55 (0.41-0.70). Bioassays involved exposure (30 $\mathrm{min}$ ) to deltamethrin, followed by recovery in seawater $(24 \mathrm{~h})$ and rating of lice as normal or affected. Dose response relationships were established for F1 females and males combined as sex differences were not significant $(P>0.05)$. Raw data are provided in Supplementary Table S11. 

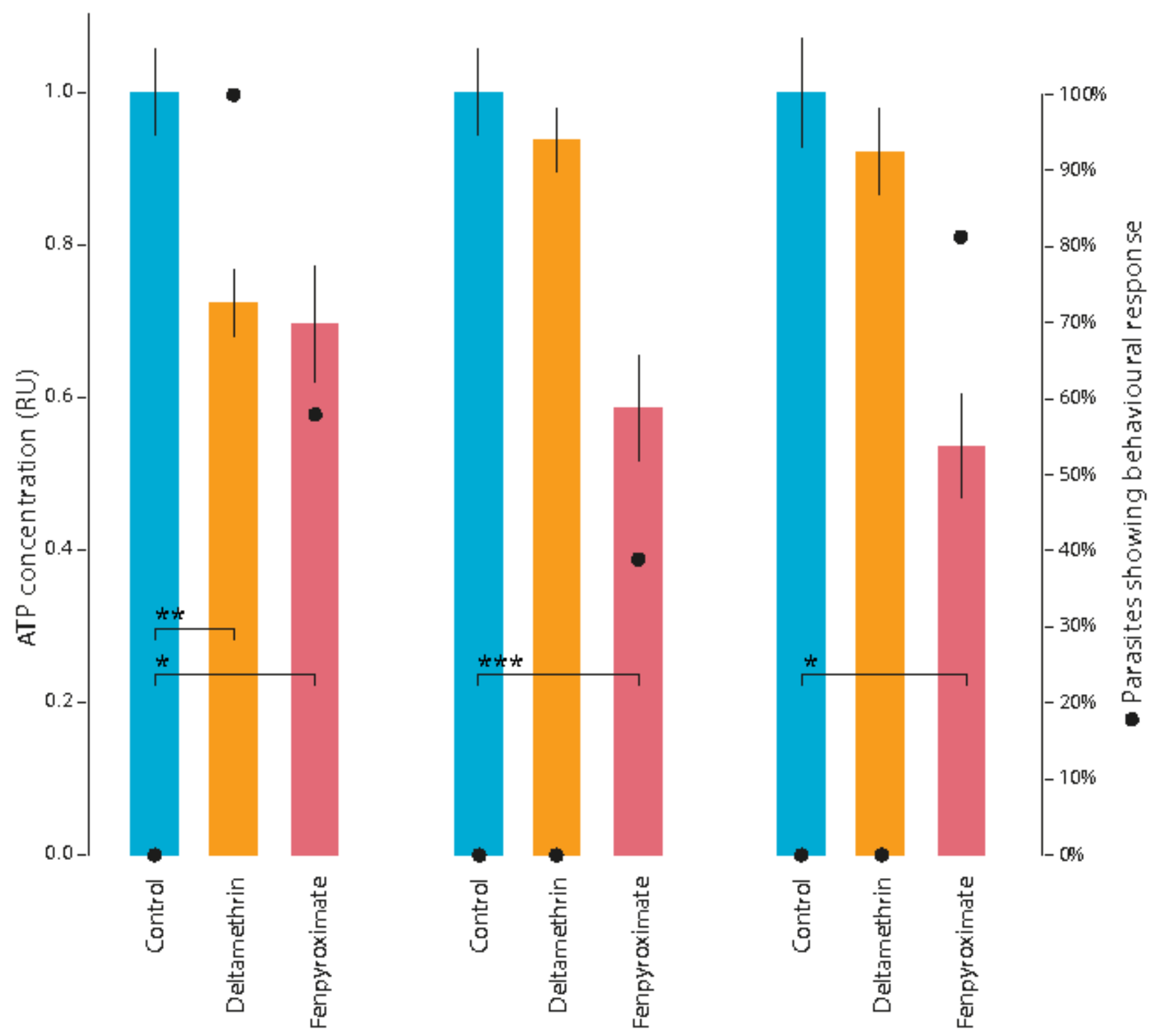

Figure 3

Effect of deltamethrin and fenpyroximate on ATP levels in L. salmonis. Male adult salmon lice of the drug susceptible strain IoA-00, the multi-resistant strain loA-02, and strain loA-10 were exposed to deltamethrin $(2 \mu \mathrm{g} / \mathrm{L})$, fenpyroximate $(100 \mu \mathrm{g} / \mathrm{L})$, or a solvent acetone control $(0.05 \% \mathrm{v} / \mathrm{v}$, control) for $300 \mathrm{~min}$ before behavioural effects were recorded (secondary y-axis; orange data points) and alive animals were sampled for whole-body ATP analysis ( $n=15$ per group). ATP concentrations in drug treated lice are expressed relative to those of the control group (relative units, $R U \pm S E$ ). Stars indicate significant differences to the

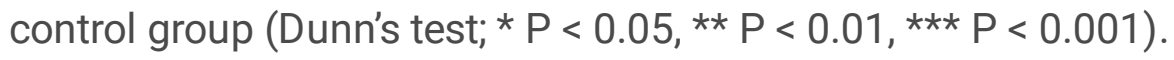

\section{Supplementary Files}

This is a list of supplementary files associated with this preprint. Click to download. 
- Supplementarymaterial.pdf

- Tables10.csv

- Tables11.csv

Page 25/25 